\title{
Dosimetric Evaluation of Body Contour Changes to Target Volumes and Organs at Risk for Cervix and Head and Neck Radiotherapy Plans
}

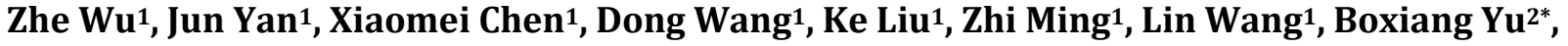 \\ Ya Pang ${ }^{*}$
}

${ }^{1}$ Department of Radiation Oncology, Zigong First People's Hospital, Zigong, China

${ }^{2}$ Institute of High Energy Physics, Chinese Academy of Sciences, Beijing, China

Email: *yubx@ihep.ac.cn, *wuzhecqu@163.com

How to cite this paper: Wu, Z., Yan, J., Chen, X.M., Wang, D., Liu, K., Ming, Z., Wang, L., Yu, B.X. and Pang, Y. (2020) Dosimetric Evaluation of Body Contour Changes to Target Volumes and Organs at Risk for Cervix and Head and Neck Radiotherapy Plans. International Journal of Medical Physics, Clinical Engineering and Radiation Oncology, 9, 96-109.

https://doi.org/10.4236/ijmpcero.2020.93010

Received: May 31, 2020

Accepted: June 19, 2020

Published: June 22, 2020

Copyright $\odot 2020$ by author(s) and Scientific Research Publishing Inc. This work is licensed under the Creative Commons Attribution International License (CC BY 4.0).

http://creativecommons.org/licenses/by/4.0/ (c) (i) Open Access

\begin{abstract}
Purpose: To investigate how much dose discrepancy would be caused by the anatomy changes during the radiotherapy (RT) course. Methods: Ten cervical cancer and ten nasopharyngeal carcinoma (NPC) CT datasets from RT patients were enrolled. The body contour from different directions changed to simulate the weight loss or gain for cervical cancer patients, who had been treated with external-beam RT using intensity-modulated radiation therapy (IMRT) or volumetric modulated arc therapy (VMAT). Moreover, the body contour from facial and shoulder superior-inferior positional change had been also assessed for NPC patients using IMRT or VMAT. The new CT (n-CT) was generated by the body contour changes with different directions based on original CT datasets. The dosimetric parameters to target volumes and organs at risk (OARs) were evaluated in Eclipse based on n-CT. Results: The target volumes and OARs were influenced by the body contour changes. Body contour expansion resulted in coverage loss, whereas body contour shrinkage increased the dose to the OARs. These findings were generally consistent for both IMRT and VMAT plans. Over a course of research, the dose to $95 \%$ of the target volumes for cervical cancer decreased by up to $2.83 \%$ per $\mathrm{cm}$ for IMRT and $2.87 \%$ per $\mathrm{cm}$ for VMAT $(\mathrm{P}<0.05)$. And the influence on $\mathrm{H} \& \mathrm{~N}$ plans was that the dose to $95 \%$ of the target volumes (low risk regions) decreased by up to $4.45 \%$ per $\mathrm{cm}$. Conclusions: The RT staff could determine whether resimulation and replaning or not according to which body contour directions were changed.
\end{abstract}

\section{Keywords}

Dosimetry, Body Contour Change, Cervical Cancer, Nasopharyngeal Carcinoma, IMRT

\footnotetext{
${ }^{\star}$ Corresponding authors.
} 


\section{Introduction}

A radiotherapy (RT) treatment starts with the acquisition of computed tomography (CT) scan, which is used to plan an individualized treatment for the patient. Highly precise RT techniques enable delivered dose in accordance with planned dose to the clinical target volumes (CTVs) and organs and risk (OARs), based on the premise that the anatomy is unchanged since the planning stage [1]. However, it takes about 1 to 2 weeks from initial CT scan to the start of RT, and a course of intensity-modulated radiation therapy (IMRT) or volumetric modulated arc therapy (VMAT) for cervical cancer or nasopharyngeal carcinoma (NPC) is typically 5 to 7 weeks [2]. During this long time, the weight change, tumor shrinkage, and shoulder position variations possibly happen, so the delivered dose to CTVs and OARs are not the same as the planned dose owing to the body contour changes.

The abdomen of cervical cancer patients usually has a lot of adipose tissue deformed with weight fluctuation. The dosimetric effects of changes in body contour, e.g., due to weight change, have been studied for head and neck [3] and prostate cancer patients [4]. Lee et al. [5] reported the weight change during RT on the development of toxicity in patients with locally advanced cervical cancer treated with IMRT. Stauch et al. [6] and Sun et al. [7] reported dosimetric effects of the body contour changes for prostate and H\&N cancer. Chow et al. [8] reported the dosimetric estimation on variations of patient size in prostate VMAT therapy. Astrid et al. [9] studied the dosimetric effects of changes in body contour for pancreatic cancer. The pieces of literature [3]-[9] showed that involuntary weight changes have a dosimetric effect on RT plans for abdominal neoplasms. Several studies show that H\&N patients' anatomy changes during the course of the treatment, and that this results in dosimetric changes from the original plans [1] [3] [7]. As a result, the patient's body contour on the treatment day could differ from the CT scan compared with cone-beam CT (CBCT) images taken on the treatment day [10]. Figure 1 illustrated a cervical cancer patient's pre-treatment and post-treatment image which is from

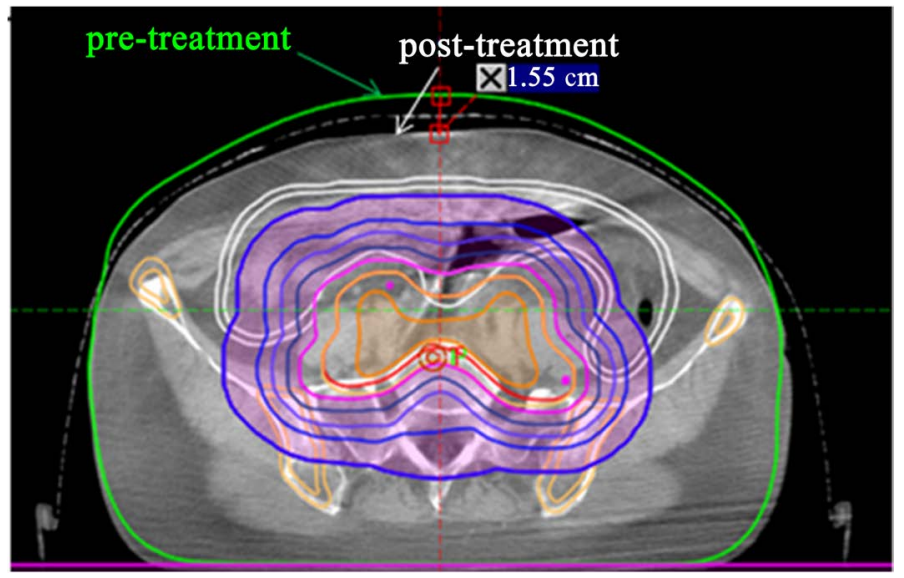

Figure 1. The body contour of pre- and post-treatment comparison. 
CBCT registration, the anterior body contour shrank $1.55 \mathrm{~cm}$ in this example. So body contour changes induced perturbations in the dose distribution, although generally only for large changes. There were no studies found about the dosimetric evaluation of target volumes and OARs from different body contour directions for tumor sites in the abdomen and pelvis, including the cervix.

The purpose of this study is to quantify for RT of cervical cancer and NPC patients the impacts on target dose coverage and OARs sparing dose parameters as a result of changes in body contour in IMRT or VMAT plans to ensure treatment efficacy, and provide a prejudgment whether further re-assessment of the plan is needed for RT staff.

\section{Materials and Methods}

\subsection{Patient Selection}

This study included ten cervical cancer patients and ten NPC patients. A total of 20 patients were randomly selected and retrospectively analyzed according to the prescription dose based on the TNM staging. The basic information of 20 selected patients was shown in Table 1. RT planning images of the 20 patients were used for this dosimetric evaluation. Their mean age of cervical cancer patients and NPC were $46.8 \pm 9.5$ and $58.3 \pm 12.4$ years old, respectively. The patients were positioned supine and immobilized with thermoplastic fixation. For each of cervix and H\&N patient, a CT image with a slice thickness of $3 \mathrm{~mm}$ was obtained by a CT simulator (Brilliance-16, Philips Medical Systems Inc., Cleveland, OH, USA).

\subsection{Body Contour Changes}

To simulate the weight's variation during the RT, different body contours change from the original CT (o-CT) were created treatment planning system (TPS, Eclipse 13.6, Varian Medical Systems Inc., Palo Alto, CA, USA). The new $\mathrm{CT}$ (n-CT) was produced by o-CT through body contour shrinkage or expansion.

For cervical patients, to simulate the weight's gain or loss, the patients' external body contours were expanded or shrunk anteriorly, posteriorly, laterally and uniformly by $1 \mathrm{~cm}$ and $2 \mathrm{~cm}$ as shown in Figure 2. For NPC patients, to simulate the weight's gain or loss, the patients' external body contours were expanded or shrunk uniformly $0.5 \mathrm{~cm}$ and $1 \mathrm{~cm}$ in the facial area (from the level of pituitary fossa to $1 \mathrm{~cm}$ inferior of the most inferior slice of mandible), and the superior-inferior direction of shoulder expanded or shrunk $1 \mathrm{~cm}, 1.5 \mathrm{~cm}$ and $2 \mathrm{~cm}$ to simulate shoulder positional changes as shown in Figure 2(e) \& Figure 2(f). The expanded tissue was assigned a CT of $0 \mathrm{HU}$ (approximately equivalent to water). The body contour changed sizes for cervical cancer and NPC was chosen based on the previous works [7] [11] [12].

\subsection{RT Plans}

All scans were exported to Eclipse for target volumes and OARs delineation and 
Table 1. Basic information of 20 selected patients.

\begin{tabular}{|c|c|c|c|c|c|}
\hline & Patients & RT Methods & Tumor Type & Prescription Dose/cGy & TNM \\
\hline & p1 & IMRT & PTV & 5000 & T3N2M0 \\
\hline & p2 & IMRT & PTV & 5000 & T3N1M0 \\
\hline & p3 & IMRT & PTV & 5000 & T3N2M0 \\
\hline & $\mathrm{p} 4$ & IMRT & PTV & 5000 & $\mathrm{~T} 2 \mathrm{~N} 2 \mathrm{M} 0$ \\
\hline Cervical & p5 & IMRT & PTV & 4600 & T1N2M0 \\
\hline \multirow[t]{9}{*}{ cancer } & p6 & VMAT & PTV & 5000 & $\mathrm{~T} 2 \mathrm{~N} 2 \mathrm{M} 0$ \\
\hline & $\mathrm{p} 7$ & VMAT & PTV & 4600 & T1N1M0 \\
\hline & $\mathrm{p} 8$ & VMAT & PTV & 5000 & T3N1M0 \\
\hline & p9 & VMAT & PTV & 4600 & T2N1M0 \\
\hline & p10 & VMAT & PTV & 5000 & T3N1M0 \\
\hline & $\mathrm{p} 1$ & IMRT & PTV1/2/3/4 & 6996/6996/6006/5412 & T3N3M0 \\
\hline & $\mathrm{p} 2$ & IMRT & PTV $1 / 2 / 3$ & 6996/6996/6006 & T3N2M0 \\
\hline & p3 & IMRT & $\mathrm{PTV} 1 / 2 / 3$ & 6996/6996/6006 & T3N2M0 \\
\hline & $\mathrm{p} 4$ & IMRT & PTV $1 / 3 / 4$ & $6996 / 6006 / 5412$ & T2N0M0 \\
\hline \multirow[t]{6}{*}{ NPC } & p5 & IMRT & $\mathrm{PTV} 1 / 2 / 3$ & 6996/6600/6006 & $\mathrm{T} 3 \mathrm{~N} 2 \mathrm{M} 0$ \\
\hline & p6 & VMAT & PTV1/3/4 & $6996 / 6006 / 5412$ & T2N0M0 \\
\hline & p7 & VMAT & PTV1/2/3/4 & 6996/6996/6006/5412 & T3N1M0 \\
\hline & $\mathrm{p} 8$ & VMAT & PTV1/2/3/4 & 6996/6996/6006/5412 & T3N1M0 \\
\hline & p9 & VMAT & $\mathrm{PTV} 1 / 2 / 3$ & 6996/6996/6006 & T1N1M0 \\
\hline & p10 & VMAT & PTV1/2/3/4 & 6996/6996/5942/5412 & T3N2M0 \\
\hline
\end{tabular}
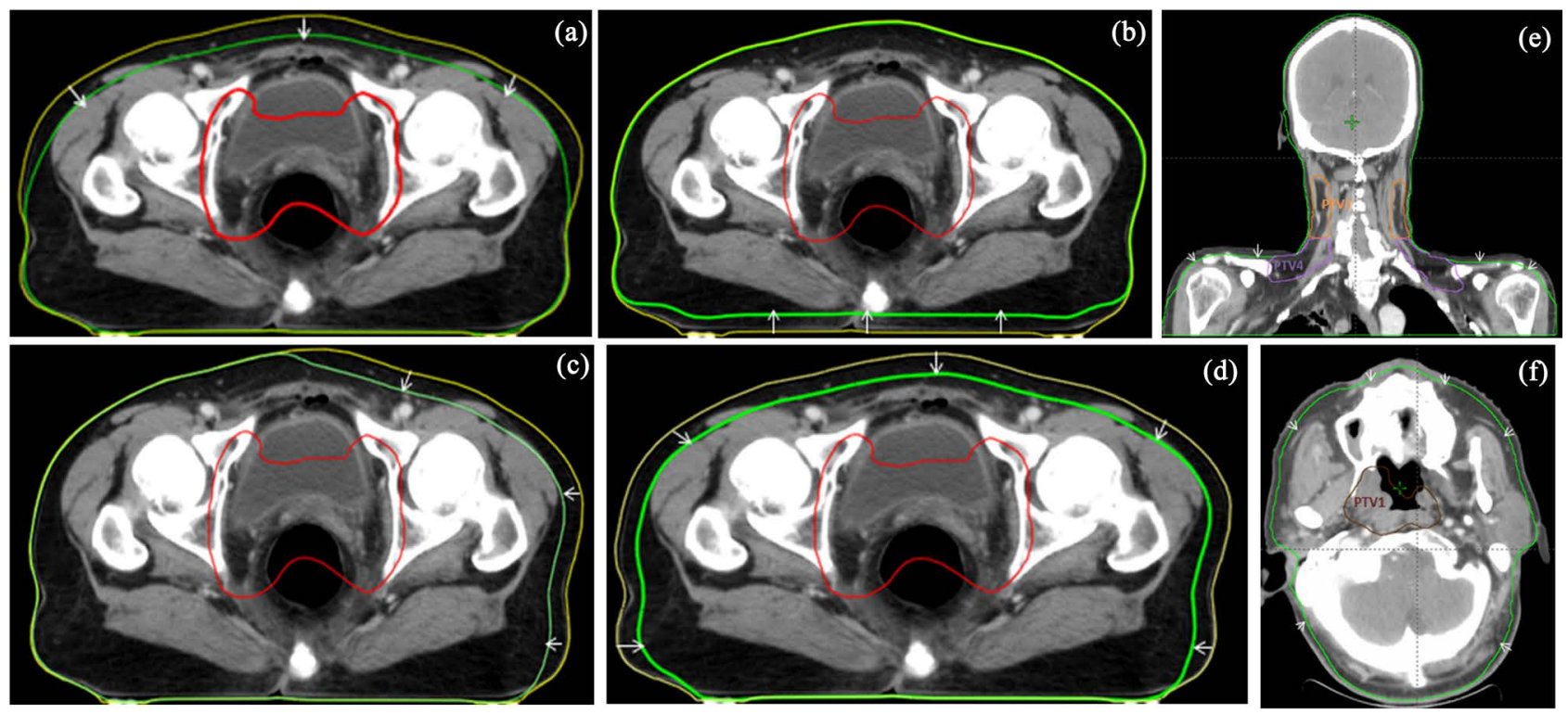

Figure 2. Body contour change model in different directions. For cervical plans: (a) anterior, (b) posterior, (c) lateral, (d) uniform directions. For NPC plans: (e) shoulder inferior, (f) facial uniform directions. 
treatment planning. The plan was delivered with 6-MV photon beams from a linear accelerator (Trilogy and Millennium 120 MLC, Varian Medical System, Palo Alto, CA, USA).

Ten cervical cancer patients had been treated with external-beam radiation therapy using IMRT $(n=5)$ and VMAT $(n=5)$. Of the 5 patients who underwent IMRT, 1 was planned to $46 \mathrm{~Gy}$ at $2.0 \mathrm{~Gy} /$ fraction, 4 was planned to $50 \mathrm{~Gy}$ at $2.0 \mathrm{~Gy} /$ fraction. Of the 5 patients who underwent VMAT, 2 were planned to $46 \mathrm{~Gy}, 3$ was planned to $50 \mathrm{~Gy}$ at $2.0 \mathrm{~Gy} /$ fraction. The IMRT plans consisted of an 8-beam arrangement, with gantry angles of $0^{\circ}, 45^{\circ}, 90^{\circ}, 135^{\circ}, 179^{\circ}, 225^{\circ}$, $270^{\circ}$, and $315^{\circ}$. The VMAT plans used two $360^{\circ}$ arcs.

Ten NPC patients' plans were also generated using IMRT $(\mathrm{n}=5)$ and VMAT $(\mathrm{n}=5)$. These 10 patients ( $\mathrm{p} 1$ to $\mathrm{p} 10)$ had already received RT with a simultaneous integrated boost to planning target volumes (PTV) including primary gross target volumes (PTV1: 66 - $70 \mathrm{~Gy}$ ), positive lymph nodal regions (PTV2: 66 - 70 Gy), high-risk metastasis regions (PTV3: 59 - 63 Gy) and low-risk metastasis regions (PTV4: 50 - 54 Gy). The IMRT plans consisted of a 9-beam arrangement, with gantry angles of $11^{\circ}, 52^{\circ}, 93^{\circ}, 134^{\circ}, 175^{\circ}, 207^{\circ}, 248^{\circ}, 289^{\circ}$, and $330^{\circ}$. The VMAT plans used two $360^{\circ}$ arcs.

The original plan (o-plan) in o-CT was copied to n-CT to recomputed (not reoptimized) to evaluate the effect of weight change on the dose distribution over numerous regions of interest. The recomputed plan was named a new plan (n-plan). The dosimetric parameters of PTV and OARs for body contour changes were statistically compared for IMRT vs VMAT plans, using a 2-sided Wilcoxon rank-sum test in SPSS (IBM Armonk, NY, USA). A p-value of 0.05 or less was considered statistically significant.

The dose to $\mathrm{D}_{95 \%}$ (the dose that covers $95 \%$ of the volumes) variation of the target was defined as $\Delta D_{95 \%}$ which could be expressed as follows:

$$
\Delta D_{95 \%}(\%)=\left(\frac{D_{95 \%, n-p l a n}}{D_{95 \%, o-p l a n}}-1\right) \times 100
$$

where, $D_{95 \%, n-p l a n}$ and $D_{95 \%, o-p l a n}$ are the $D_{95 \%}$ of the n-plan and o-plan, respectively.

The maximal dose variation of OARs was defined as $\Delta D_{\max }$ which could be expressed as follows:

$$
\Delta D_{\max }=D_{\max , n-p l a n}-D_{\max , 0-p l a n}
$$

where, $D_{\max , n-p l a n}$ and $D_{\max , o-p l a n}$ is the $D_{\max }$ of the n-plan and o-plan, respectively.

\section{Results}

\subsection{Target Volumes}

Figure 3 shows the $\Delta D_{95 \%}$ variation for ten cervical plans as body contour change in anterior $(\mathrm{A})$, posterior $(\mathrm{P})$, uniform $(\mathrm{U})$ and lateral $(\mathrm{L})$ direction. We have assigned a short name to the body contour change. + and - means contour expansion and shrinkage, respectively. For instance, $\mathrm{A}+2$ means the body contour expanded $2 \mathrm{~cm}$ in an anterior direction and $\mathrm{A}-2$ means body contour shrank 

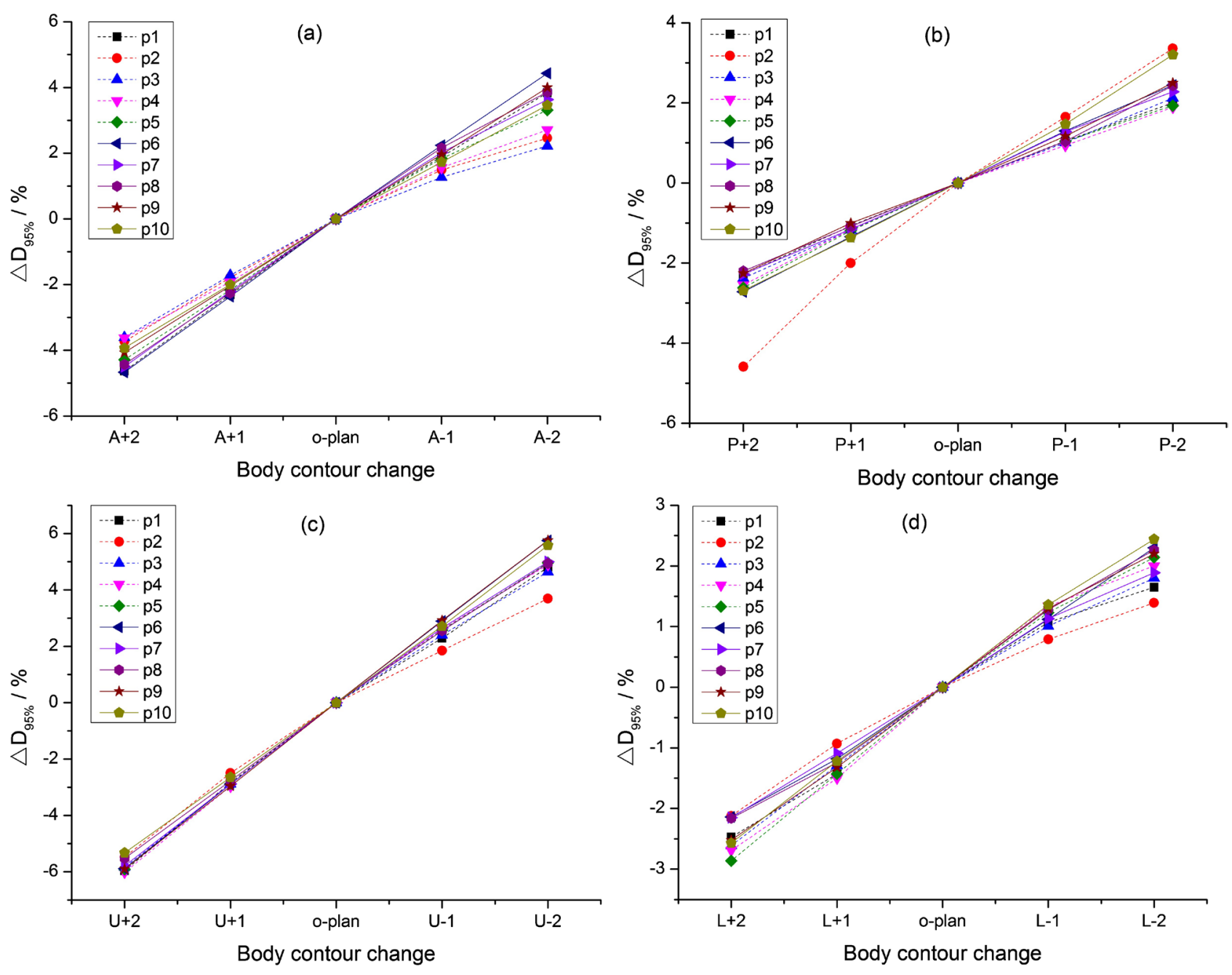

Figure 3. Variation in $\Delta D_{95 \%}$ of PTV due to body contour change in $\mathrm{A}, \mathrm{P}, \mathrm{U}, \mathrm{L}$ direction for cervical plans.

$2 \mathrm{~cm}$ in the anterior direction, similarly for other directions. The $\Delta D_{95 \%}$ of target volumes increased near linearly as body contour shrank. The $\Delta D_{95 \%}$ variations were fitted linearly. The mean slopes of the lines were $-1.98 \% \pm 0.1 \%,-1.21 \% \pm$ $0.4 \%,-2.83 \% \pm 0.5 \%$ and $-1.3 \% \pm 0.2 \%$ per $\mathrm{cm}$ for IMRT in A, P, U and L direction expanding, respectively. And the mean slopes of the lines were $-2.16 \% \pm$ $0.1 \%,-1.25 \% \pm 0.6 \%,-2.87 \% \pm 0.3 \%$ and $-1.18 \% \pm 0.3 \%$ per $\mathrm{cm}$ for VMAT. The difference between IMRT and VMAT was significant $(\mathrm{p}<0.05)$ in A, U, L directions. For both IMRT and VMAT plans, the anterior region $\Delta D_{95 \%}$ showed larger variations than do in the posterior region, this was mainly because the anterior body contour changed length is longer than the posterior body contour change. In $\mathrm{A}$ and $\mathrm{U}$ direction, the $\Delta D_{95 \%}$ for VMAT was overall larger than that for IMRT, which is mainly because in VMAT plans the dose is delivered by two full arcs with multileaf collimator modulation and the IMRT plans the dose is delivered in an 8-beam arrangement. Whereas in L direction, the $\Delta D_{95 \%}$ shows slightly larger than that for VMAT, probably because in IMRT plans, the gantry angle of $45^{\circ}, 90^{\circ}$ and $135^{\circ}$ had a greater influence than VMAT plans during the 

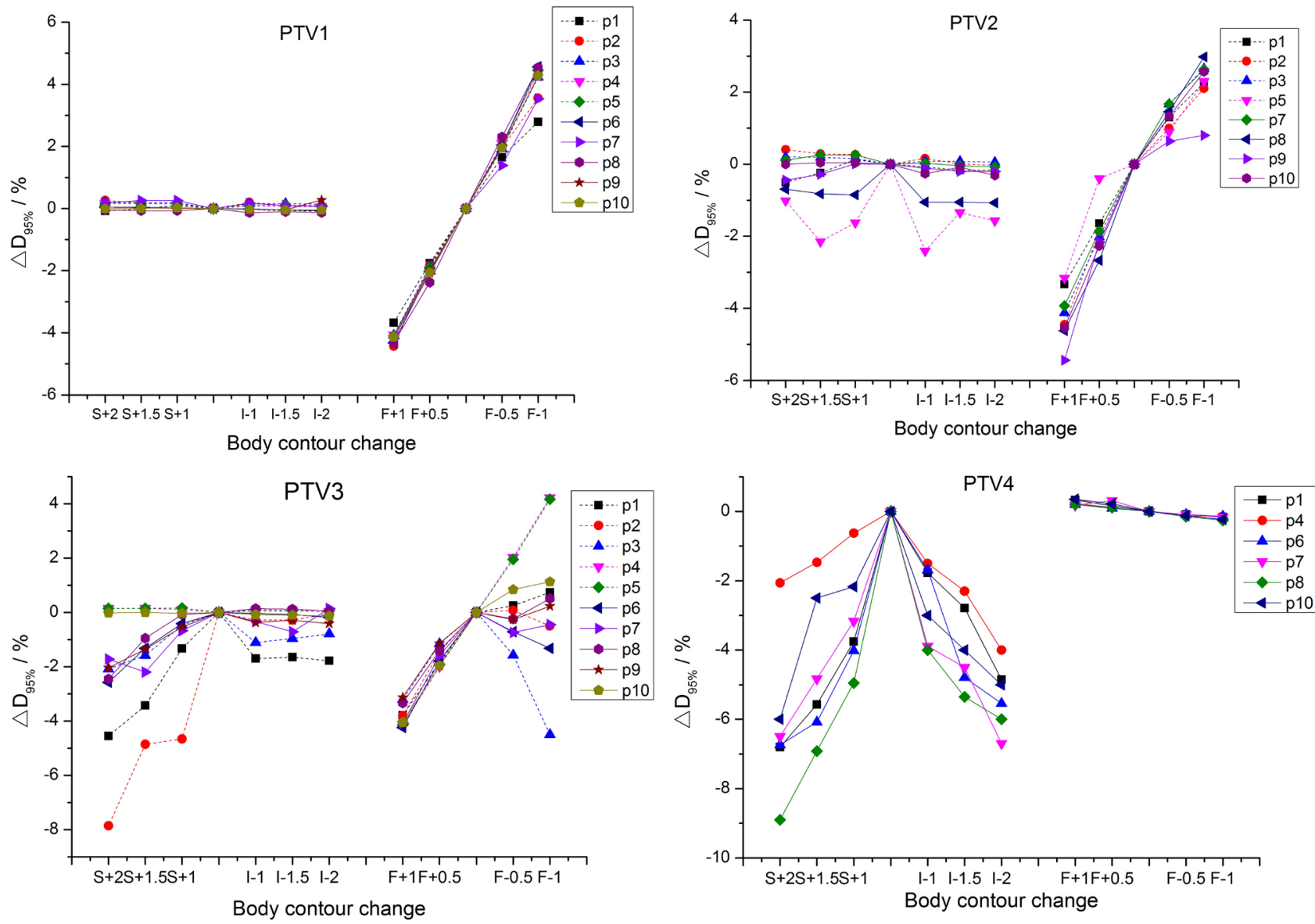

p4, p6: without PTV2; p2, p3, p5, p9: without PTV4.

Figure 4. Variation in $\Delta D_{95 \%}$ of PTV due to body contour change in S, I, F direction for NPC plans. 
C5-C6 region in p6, p7, p8, so the shoulder body contour expansion had larger influence to PTV3, particularly those with the lower neck region. The shoulder body contour shrinkage had a relatively smaller influence on PTV3. The facial body contour expansion led to $\Delta D_{95 \%}$ loss in the 10 plans, but the facial body contour shrinkage had both $\Delta D_{95 \%}$ loss and gain. For both cervix and NPC patients, p1, p $2 \cdots$ p 5 are IMRT plans, and p6, p $7 \cdots$ p10 are VMAT plans. For both IMRT and VMAT plans, shoulder body contour change in the superior-inferior directions resulted in the greatest loss of coverage for PTV4 and up to $4.45 \% / \mathrm{cm}$ (p8), because the PTV4 is lower neck target (from C6 to T2 vertebrae). The facial body contour change did not show a large loss in PTV4 because PTV4 was far enough from the facial region.

\subsection{OARs Sparing}

In addition to decreasing the dose to the target volumes, body contour change also has the potential to increase the dose to OARs. The change in dose to the bladder, rectum, small bowel for cervical patients and brain stem and the spinal cord for NPC patients in each direction change was evaluated in Eclipse for both IMRT and VMAT plans.

For the ten selected cervical patients, the rectum, bladder and small bowel were investigated from the dose-volume histogram. Figures 5(a)-(d) show the $\Delta D_{50 \%}$ changes for rectum in A, P, U, L directions, respectively. The $\Delta D_{50 \%}$ were $1.86 \% \pm 0.16 \%, 0.96 \% \pm 0.31 \%, 2.83 \% \pm 0.31 \%, 1.32 \% \pm 0.23 \%$ per $\mathrm{cm}$ for IMRT and $1.49 \% \pm 0.21 \%, 1.86 \% \pm 0.59 \%, 2.40 \% \pm 0.24 \%, 1.25 \% \pm 0.16 \%$ per $\mathrm{cm}$ for VMAT in A, P, U and L directions shrinkage, respectively. The difference between IMRT and VMAT was significant $(\mathrm{p}<0.05)$ in all directions. Only in the $\mathrm{P}$ direction, the $\Delta \mathrm{D}_{50 \%}$ for VMAT was larger than that for IMRT, which is mainly because the rectum is close to the posterior body contour and the range of beam path length affected by body contour change and the relative portion of the dose delivered in the corresponding direction. Figures $6(\mathrm{a})$-(d) show the $\Delta \mathrm{D}_{50 \%}$ changes for bladder in $\mathrm{A}, \mathrm{P}, \mathrm{U}, \mathrm{L}$ directions, respectively. The $\Delta \mathrm{D}_{50 \%}$ were $2.06 \%$ $\pm 0.48 \%, 1.24 \% \pm 0.53 \%, 2.32 \% \pm 0.41 \%, 1.12 \% \pm 0.21 \%$ per $\mathrm{cm}$ for IMRT and $2.41 \% \pm 0.29 \%, 1.14 \% \pm 0.24 \%, 2.98 \% \pm 0.32 \%, 1.31 \% \pm 0.16 \%$ per $\mathrm{cm}$ for VMAT in $\mathrm{A}, \mathrm{P}, \mathrm{U}$ and $\mathrm{L}$ direction shrinkage, respectively $(\mathrm{P}<0.05)$. The results of $\Delta D_{50 \%}$ for bladder were opposite to the $\Delta D_{50 \%}$ for rectum for IMRT and VMAT in A, P, U, L directions, which is mainly because the engorged bladder is enough larger than the rectum in volume which involved the target area. The dose is delivered when the gantry was moving for VMAT plans. So the bladder $\Delta D_{50 \%}$ for VMAT shows overall larger than it in the rectum for IMRT except for $\mathrm{P}$ direction. Figure 7 shows the small bowel $\Delta D_{\max }$ variation. The body contour expansion leads to $D_{\max }$ decrease and shrinkage leads to dose increase for small bowel. The $D_{\max }$ of small bowel increased by up to $391 \mathrm{cGy}, 163 \mathrm{cGy}$, 439 cGy, 276 cGy for IMRT and 295 cGy, 378 cGy, 348 cGy, 188 cGy for VMAT in A-2, P-2, U-2, and L-2 direction, respectively. 

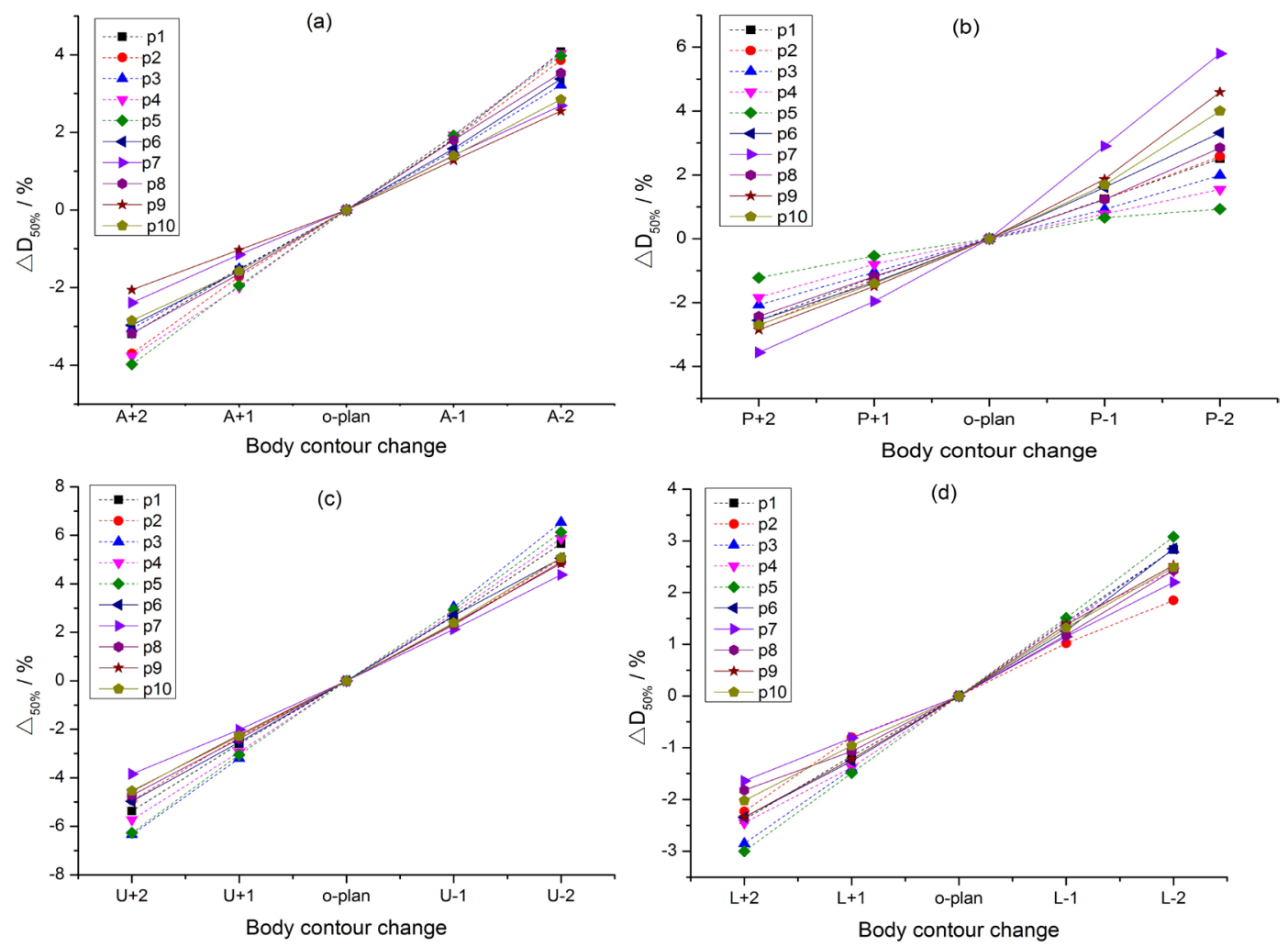

Figure 5. Variation in $\Delta \mathrm{D}_{50 \%}$ of the rectum due to body contour change in $\mathrm{A}, \mathrm{P}, \mathrm{U}, \mathrm{L}$ directions for cervical plans.
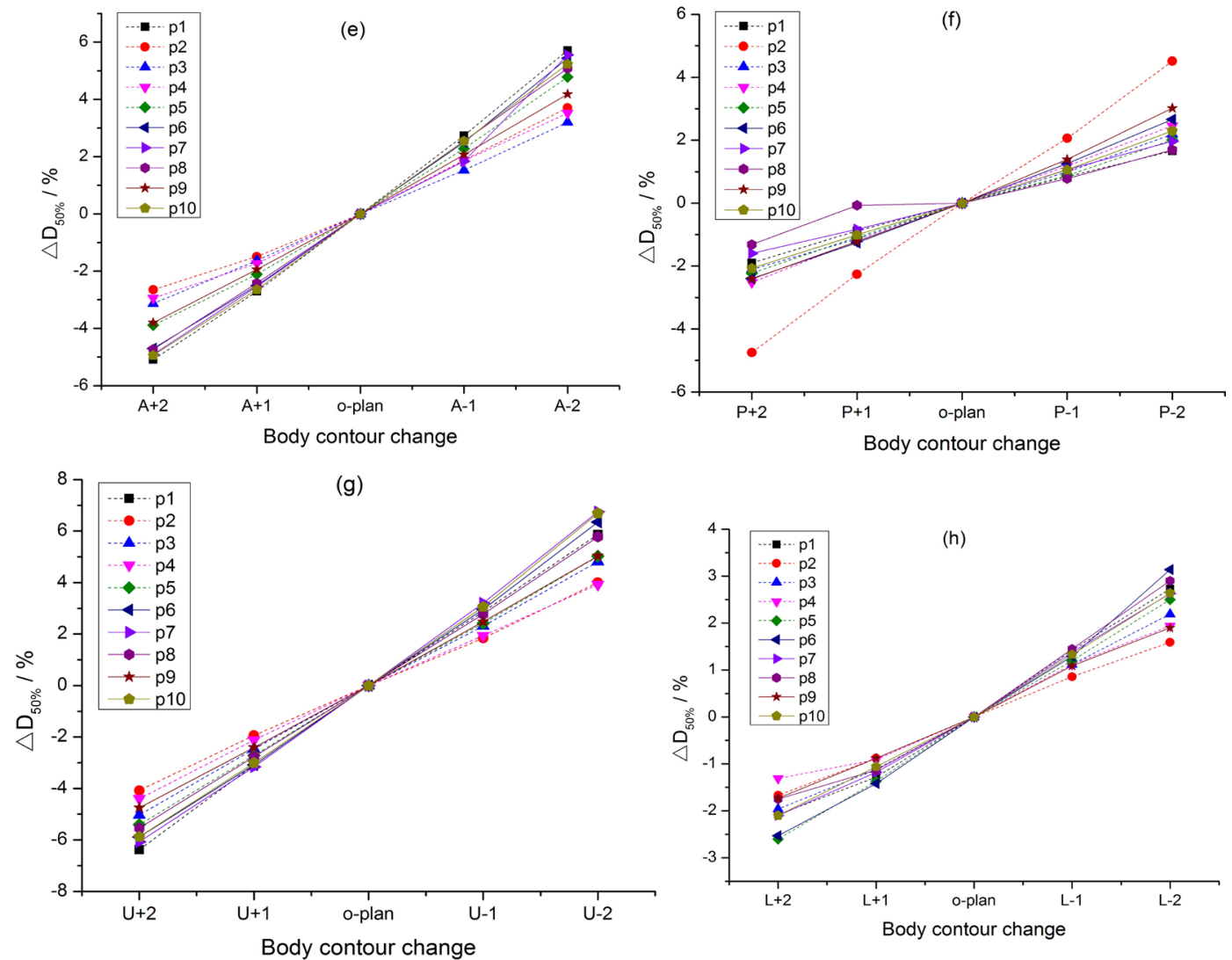

Figure 6. Variation in $\Delta \mathrm{D}_{50 \%}$ of the bladder due to body contour change in $\mathrm{A}, \mathrm{P}, \mathrm{U}, \mathrm{L}$ directions for cervical plans. 
For the ten selected NPC patients, the $D_{\max }$ change to the brain stem and spinal cord was displayed in Figure 8. The $\Delta D_{\max }$ of the brain stem of all of the examined dosimetric cases was up to $195 \mathrm{cGy}$ for IMRT and $210 \mathrm{cGy}$ for VMAT in F-1 direction, and the $\Delta D_{\max }$ of the spinal cord increased by up to 209 cGy for IMRT and $628 \mathrm{cGy}$ for VMAT in S-2 direction.

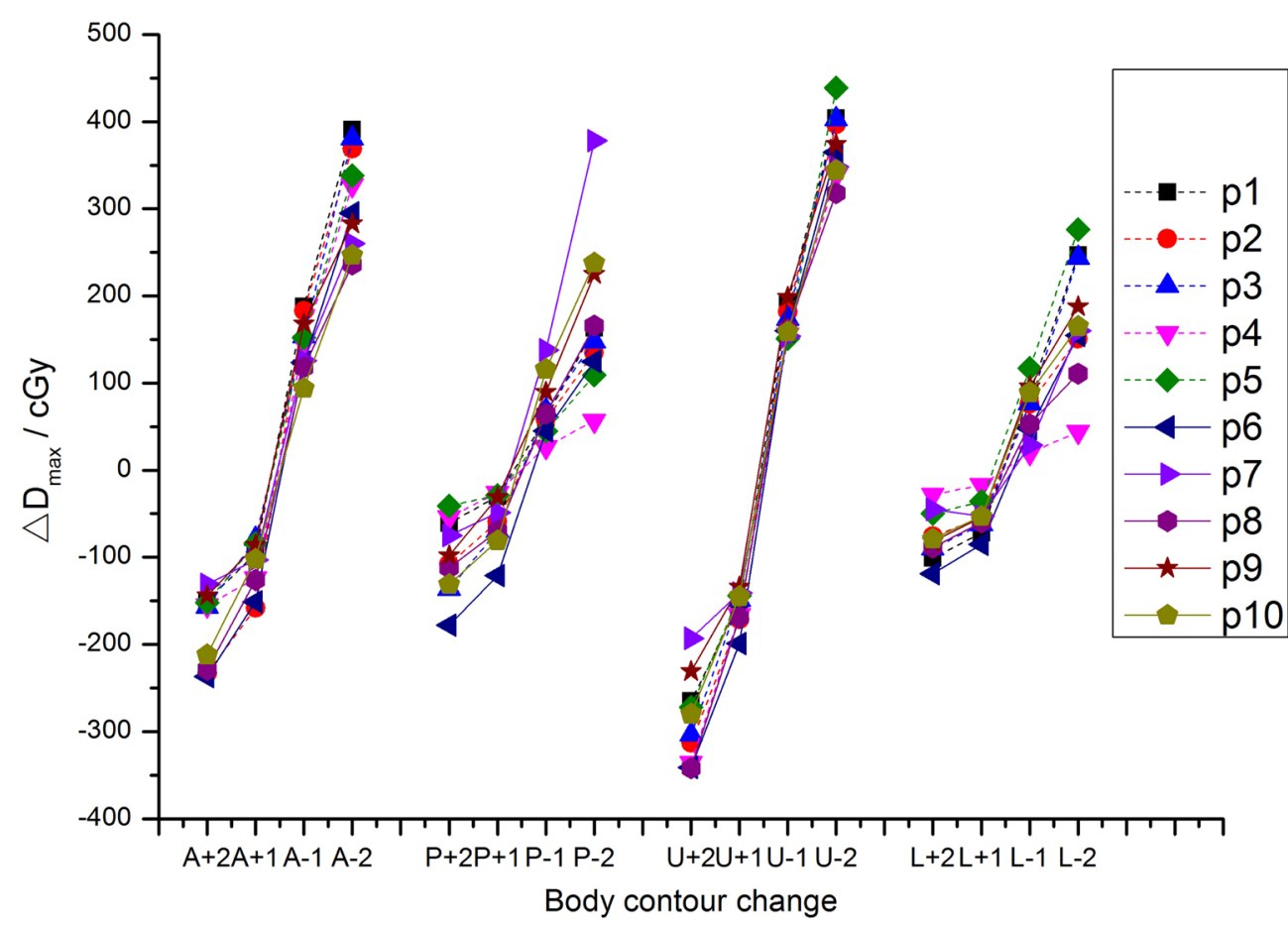

Figure 7. Variation in $\Delta D_{\max }$ of small bowel due to body contour change in $\mathrm{A}, \mathrm{P}, \mathrm{U}, \mathrm{L}$ directions for cervical plans.

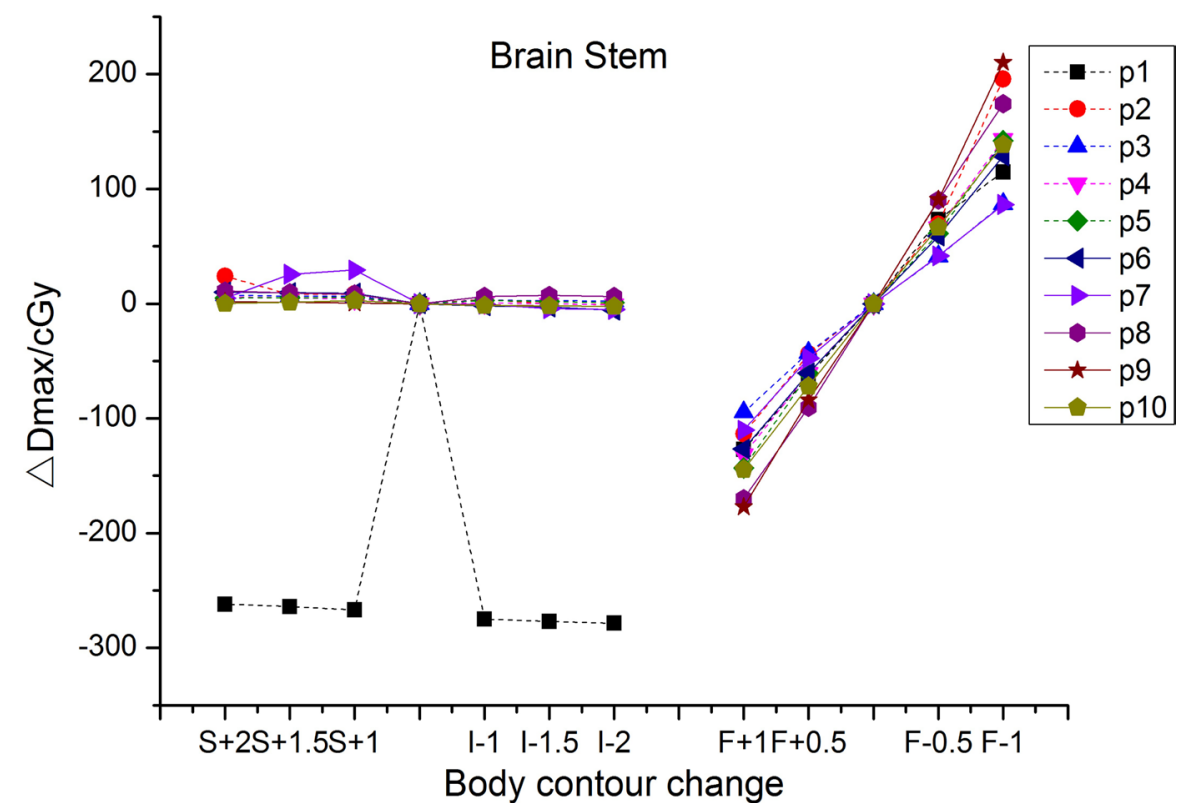

Figure 8. Variation in $\Delta D_{\max }$ of the brain stem and spinal cord due to body contour change in S, I, F directions for NPC plans. 


\section{Discussion}

Accurate dose delivery to target volumes and organs at risk (OARs) is essential to ensure the radiotherapeutic effect and minimize the risk of normal tissue toxicity, whereas weight fluctuation frequently occurs during the whole radiotherapy course, which may cause the body contour changes to effect on treatment accuracy. Therefore, we evaluated the impacts of body contour changes to target volumes and OARs in the radiotherapy plans of cervical cancer and head and neck tumors. A course of RT is typically several weeks. During the long period from the CT scans to RT finish, the weight change for abdominal and $\mathrm{H} \& \mathrm{~N}$ patients was reported in previous literature [13] [14] [15] [16]. There is evidence showing that weight change is correlated with external contour changes [17].

Booth et al. [18] reported that $68 \%$ of the 198 analyzed СВCT images from 19 prostate patients were in the range of $0-1 \mathrm{~cm}, 28 \% 1-2 \mathrm{~cm}$, and $4 \%>2 \mathrm{~cm}$ with deviations occurring mostly in the postero-lateral direction. Chow et al. [13] studied body contour shrunk by $0.5,1,1.5,2 \mathrm{~cm}$ in anterior, left and right direction for five patients' IMRT and VMAT prostate plans. Sun et al. [7] reported that prostate patients who have body contour change less than $2 \mathrm{~cm}$ at a single side or less than $1 \mathrm{~cm}$ uniformly are unlikely to need further assessment. For H\&N patients, Chen et al. [14] reported 25 NPC patients shrank the external contours with different margins $(2,3$, and $5 \mathrm{~mm}$ ). Our study illustrated the detailed dosimetry of PTV and OARs for cervical cancer and NPC in different body contour changes based on previous works [4] [7].

Currently, IMRT or VMAT are widely used for planning to treat patients with cervical cancer and NPC, which the dosimetric effect of body contour change in different directions is still unknown. Our contour-size change model is consistent with that used in a recent study by Sun et al. [7], in which the contour-size effect was assessed for VMAT only. Also, Sun et al. only evaluated the dose percentage and isodose line shift whereas our works focus on the dose variations of $D_{95 \%}$ for whole PTV and $D_{50 \%}$ or $D_{\max }$ for OARs. Another discrepancy between our means with Sun et al. is that the different PTVs with simultaneous integrated boost for $\mathrm{H} \& \mathrm{~N}$ patients were discussed in this paper. This study showed that the body contour shrinkage (expansion) caused the dose increase (decrease) to PTV or OARs and this finding matches the results from the study published by Pair et al. [4]. This paper showed the cervical patients' $D_{95 \%}$ of target volumes decreased by up to $1.98 \%, 1.21 \%, 2.87 \%, 1.3 \%$ per $\mathrm{cm}$ for IMRT and $2.16 \%, 1.38 \%, 2.83 \%$ and $1.18 \%$ for VMAT in A, P, U and L direction expanding, respectively. Pair et al. [4] reported that the prostate patients' target mean dose decreased by $2.9 \%$ per $\mathrm{cm}$ for IMRT and by $3.6 \%$ for VMAT in $\mathrm{U}$ direction. The result of IMRT plans is larger than do VMAT plans in our research which was mainly because the IMRT Gantry angle arrangement is different. In Pair's [4] study, the change of $D_{10 \%}$ for rectum and bladder were $2.8 \%$ to $3.5 \%$ which were close to our study that the $D_{50 \%}$ for rectum and bladder were increased by up to $2.98 \%$.

For $\mathrm{H} \& \mathrm{~N}$ plans, we studied the body contour changes in facial area and 
shoulder based on the previous study by Sun et al. [7] and Neubauer et al. [19]. Neubauer et al. [19] examined ten patients and 243 CTs, and found that $2 \%$ of shoulder shifts were greater than $1 \mathrm{~cm}$. Noble et al. [1] measured lateral neck diameter which is $175 \mathrm{~mm}$ on the first day and $162 \mathrm{~mm}$ on the final treatment day. In our study, we chose shoulder changed $1 \mathrm{~cm}$ and $2 \mathrm{~cm}$ in S-I directions and $0.5 \mathrm{~cm}$ and $1 \mathrm{~cm}$ in the facial area. Chen et al. [14] shrank external contour with different margins $\left(2,3\right.$, and $5 \mathrm{~mm}$ ) and reported that the $\mathrm{D}_{95 \%}$ of PTV1 was increased by $1.9 \%$ to $2.9 \%$, which was similar to our result $1.95 \% \pm 0.58 \% / 5 \mathrm{~mm}$. The PTV3/4 in Figure 4 shows relative irregular changes for $\mathrm{D}_{95 \%}$ compared with PTV1/2. The PTV3/4 extended outside of the shrunk body contour, so the volumes within the new contour were affected by the build-up effect. Zhao et al. [20] found an increase in the maximum dose to the spinal cord and brainstem volumes of $560 \mathrm{cGy}$ and $250 \mathrm{cGy}$, respectively, by comparing repeat CT imaging to the dose distribution on the original planning CT. Wang et al. [21] reported the NPC repeated CT scan after 18 fractions, the mean volume of the left and right parotid decreased $6.19 \mathrm{~mL}$ and $6.44 \mathrm{~mL}$, and the center of $\mathrm{C} 2$ vertebral body slices contracted with the mean contraction of $8.2 \mathrm{~mm}, 9.4 \mathrm{~mm}$, and 7.6 $\mathrm{mm}$ while the maximum dose to the brain stem and spinal cord increased by 0.08 to $6.51 \mathrm{~Gy}$ and 0.05 to $7.8 \mathrm{~Gy}$. Our study showed that the $\Delta D_{\max }$ of the brain stem of all of the examined dosimetric cases was up to $195 \mathrm{cGy}$ for IMRT and 210 cGy for VMAT in F-1 direction, and the $\Delta D_{\max }$ of the spinal cord increased by up to $209 \mathrm{cGy}$ for IMRT and $628 \mathrm{cGy}$ for VMAT in S-2 direction. The dosimetrists need to take the dosimetric changes into account during the RT plan design.

The factors which affect the dose to target volumes and OARs were anatomy and setup error, this paper studied dose discrepancy which resulted from the anatomy change. The limitation of this study was that the location, geometry, and size of the tumor, OARs may change during the RT period. Moreover, the patients' body contour change may not be as regular in real clinical status. These situations were not discussed in this study. The body contour changed methods in this work have been widely reported in previous literature [4] [7] [8] [13] [14]. It is a simplified way but good choice to predict such complicated dosimetric problems. Therefore, the RT staff could make a preliminary judgment of dosimetric parameters induced by body contour changes for cervical cancer and NPC patients based on the findings throughout this work.

\section{Conclusion}

The dosimetric evaluation of body contour changes to PTV and OARs for cervical cancer and NPC plans was studied. The body contour shrinkage or expansion affects the IMRT and VMAT dose delivery. The body contour changes may impact the dosimetry of the PTV and OARs to a different extent, depending on the directions and magnitude of the body contour changes. The RT staff could determine whether resimulation and replanning or not according to which body 
contour directions were changed.

\section{Acknowledgements}

This work was supported by National Natural Science Foundation of China (Grant No. 11575221), National Key Research and Development Program of China (2018YFA0404300).

\section{Conflicts of Interest}

The authors declare no conflicts of interest regarding the publication of this paper.

\section{References}

[1] Noble, D.J., Ping-Lin, Y., Seah, S.Y.K., et al. (2019) Anatomical Change during Radiotherapy for Head and Neck Cancer, and Its Effect on Delivered Dose to the Spinal Cord. Radiotherapy and Oncology, 130, 32-38.

https://doi.org/10.1016/j.radonc.2018.07.009

[2] Gowda, R.V., Henk, J.M., Mais, K.L., et al. (2003) Three Weeks Radiotherapy for T1 Glottic Cancer: The Christie and Royal Marsden Hospital Experience. Radiotherapy and Oncology, 68, 105-111. https://doi.org/10.1016/S0167-8140(03)00059-8

[3] Cheng, H.C.Y., Wu, V.W.C., Ngan, R.K.C., et al. (2012) A Prospective Study on Volumetric and Dosimetric Changes during Intensity-Modulated Radiotherapy for Nasopharyngeal Carcinoma Patients. Radiotherapy and Oncology, 104, 317-323. https://doi.org/10.1016/j.radonc.2012.03.013

[4] Pair, M.L., Du, W., Rojas, H.D., et al. (2013) Dosimetric Effects of Weight Loss or Gain during Volumetric Modulated Arc Therapy and Intensity-Modulated Radiation Therapy for Prostate Cancer. Medical Dosimetry, 38, 251-254. https://doi.org/10.1016/j.meddos.2013.02.004

[5] Lee, J., Chang, C.L., Lin, J.B., et al. (2018) The Effect of Body Mass Index and Weight Change on Late Gastrointestinal Toxicity in Locally Advanced Cervical Cancer Treated with Intensity-Modulated Radiotherapy. International Journal of Gynecological Cancer, 28, 1377-1386. https://doi.org/10.1097/IGC.0000000000001312

[6] Stauch, Z., Zoller, W., Tedrick, K., et al. (2020) An Evaluation of Adaptive Planning by Assessing the Dosimetric Impact of Weight Loss throughout the Course of Radiotherapy in Bilateral Treatment of Head and Neck Cancer Patients. Medical Dosimetry, 45, 52-59. https://doi.org/10.1016/j.meddos.2019.05.003

[7] Sun, L., Kirkby, C. and Smith, W. (2019) Dosimetric Effect of Body Contour Changes for Prostate and Head and Neck Volumetric Modulated Arc Therapy Plans. Journal of Applied Clinical Medical Physics, 20, 115-124. https://doi.org/10.1002/acm2.12571

[8] Chow, J.C.L. and Jiang, R. (2013) Dosimetry Estimation on Variations of Patient Size in Prostate Volumetric Modulated Arc Therapy. Medical Dosimetry, 38, 42-47. https://doi.org/10.1016/j.meddos.2012.05.005

[9] Astrid, V.D.H., Houweling, A.C., Van, T.G., et al. (2017) Dosimetric Effects of Anatomical Changes during Fractionated Photon Radiation Therapy in Pancreatic Cancer Patients. Journal of Applied Clinical Medical Physics, 18, 142-151. https://doi.org/10.1002/acm2.12199 
[10] Lin, L.L., Hertan, L., Rengan, R., et al. (2012) Effect of Body Mass Index on Magnitude of Setup Errors in Patients Treated with Adjuvant Radiotherapy for Endometrial Cancer with Daily Image Guidance. International Journal of Radiation Oncology Biology Physics, 83, 670-675. https://doi.org/10.1016/j.ijrobp.2011.07.026

[11] Stanley, K., Eade, T., Kneebone, A. and Booth, J.T. (2015) Investigation of an Adaptive Treatment Regime for Prostate Radiation Therapy. Practical Radiation Oncology, 5, e23-e29. https://doi.org/10.1016/j.prro.2014.03.015

[12] Tsai, Y., Cheng, S. and Huang, C. (2015) EP-1136 Adaptive Radiotherapy for Nasopharyngeal Carcinoma. Radiotherapy and Oncology, 115, S618. https://doi.org/10.1016/S0167-8140(15)41128-4

[13] Chow, J.C.L. and Jiang, R. (2013) Comparison of Dosimetric Variation between Prostate IMRT and VMAT Due to Patient's Weight Loss: Patient and Phantom Study. Reports of Practical Oncology \& Radiotherapy, 18, 272-278. https://doi.org/10.1016/j.rpor.2013.05.003

[14] Chen, C., Fei, Z., Chen, L., et al. (2014) Will Weight Loss Cause Significant Dosimetric Changes of Target Volumes and Organs at Risk in Nasopharyngeal Carcinoma Treated with Intensity-Modulated Radiation Therapy? Medical Dosimetry, 39, 34-37. https://doi.org/10.1016/j.meddos.2013.09.002

[15] Brouwer, C.L., Steenbakkers, R.J.H.M., Langendijk, J.A., et al. (2015) Identifying Patients Who May Benefit from Adaptive Radiotherapy: Does the Literature on Anatomic and Dosimetric Changes in Head and Neck Organs at Risk during Radiotherapy Provide Information to Help? Radiotherapy and Oncology, 115, 285-294. https://doi.org/10.1016/j.radonc.2015.05.018

[16] Ottosson, S., Zackrisson, B., Kjellen, E., Nilsson, P. and Laurell, G. (2013) Weight Loss in Patients with Head and Neck Cancer during and after Conventional and Accelerated Radiotherapy. Acta Oncologica, 52, 711-718. https://doi.org/10.3109/0284186X.2012.731524

[17] Ahn, P.H., Chen, C.C., Ahn, A.I., et al. (2011) Adaptive Planning in Intensity-Modulated Radiation Therapy for Head and Neck Cancers: Single-Institution Experience and Clinical Implications. International Journal of Radiation Oncology - Biology • Physics, 80, 677-685. https://doi.org/10.1016/j.ijrobp.2010.03.014

[18] Booth, J., Eade, T. and Forde, E. (2010) SU-GG-J-68: Significant Dosimetric Impact of Variable FSDs during Prostate IMRT: The Role of CBCT. Medical Physics, 37, 3160-3161. https://doi.org/10.1118/1.3468292

[19] Neubauer, E., Dong, L., Followill, D.S., et al. (2012) Assessment of Shoulder Position Variation and Its Impact on IMRT and VMAT Doses for Head and Neck Cancer. Radiation Oncology, 7, 19. https://doi.org/10.1186/1748-717X-7-19

[20] Zhao, L., Wan, Q., Zhou, Y., et al. (2011) The Role of Replanning in Fractionated Intensity Modulated Radiotherapy for Nasopharyngeal Carcinoma. Radiotherapy and Oncology, 99, 23-27. https://doi.org/10.1016/j.radonc.2010.10.009

[21] Wang, X., Lu, J., Xiong, A., et al. (2010) Anatomic and Dosimetric Changes during the Treatment of Intensity-Modulated Radiotherapy for Locally Advanced Nasopharyngeal Carcinoma. Medical Dosimetry, 35, 151-157.

https://doi.org/10.1016/j.meddos.2009.06.007 